\title{
Dabigatran for prophylaxis of thromboembolic events in patients with atrial fibrillation
}

This article was published in the following Dove Press journal:

Research Reports in Clinical Cardiology

17 January 2012

Number of times this article has been viewed

Joachim R Ehrlich'

Matthias Hammwöhner ${ }^{3}$

Andreas Goette ${ }^{2,3}$

'Deutsche Klinik für Diagnostik, Department of Cardiology,

Wiesbaden, ${ }^{2}$ Working Group, Molecular Electrophysiology,

University Hospital Magdeburg,

${ }^{3}$ Department of Cardiology and

Intensive Care Medicine,

St Vincenz-Hospital Paderborn,

Germany
Correspondence: Andreas Goette Department of Cardiology and Intensive Care Medicine, St Vincenz Hospital Paderborn $\mathrm{GmbH}$, Am Busdorf 2, 33098 Paderborn, Germany

$\mathrm{Tel}+49525 \mathrm{I} 86165 \mathrm{I}$

Fax +49525I 861652

Email andreas.goette@med.ovgu.de
Abstract: Atrial fibrillation is the most common clinically relevant cardiac arrhythmia. Prevalence and incidence rates are constantly rising with advancing population age. A severe complication of untreated atrial fibrillation is thrombus formation in the left atrial appendage with consecutive peripheral thromboembolism. Thus, atrial fibrillation is a major contributor to thromboembolic events, especially in the older population. Depending on the $\mathrm{CHADS}_{2}$ risk score for thromboembolic events, oral anticoagulation therapy with vitamin $\mathrm{K}$ antagonists is currently the treatment of choice for the prevention of thromboembolism, including apoplectic strokes. However, due to the drawbacks of current anticoagulation therapy, new substances for oral anticoagulation therapy are currently being evaluated in various clinical studies. This article will provide an up to date overview of orally active compounds for the future treatment of atrial fibrillation using the direct thrombin inhibitor, dabigatran.

Keywords: atrial fibrillation, anticoagulation, pathophysiology, pharmacology, stroke

\section{Introduction}

Atrial fibrillation is the most common cardiac arrhythmia with an increased prevalence in the elderly. ${ }^{1}$ During atrial fibrillation, chaotic electrical propagation prevents coordinated contraction of the atria. The resulting decreased flow velocity promotes blood stasis. It has been shown that the atrial endocardium and myocardium are involved in numerous electrical and structural remodeling processes during atrial fibrillation. Clinically, remodeling processes and activation of the clotting system may lead to the most common and severe complication of atrial fibrillation, ie, thromboembolic stroke. ${ }^{2-6}$

Strategies to prevent this potentially disastrous clinical event have focused on inhibition of the coagulation cascade with anticoagulants (Figure 1). ${ }^{4-6}$ Until now, oral therapy with vitamin $\mathrm{K}$ antagonists has been considered to be the best therapy for patients with atrial fibrillation at increased risk for stroke. ${ }^{4-8}$ Nevertheless, the novel anticoagulant dabigatran has been shown to be superior to warfarin. Therefore, this review will summarize the pharmacological and clinical aspects of dabigatran.

\section{Pathophysiology of atrial fibrillation}

Atrial fibrillation is known to induce significant electrophysiological and structural changes in cardiac tissue, which contribute to the persistence of atrial fibrillation. ${ }^{7,9,10}$ The overall pathophysiological mechanism for electrophysiological changes in atrial myocytes has been termed "electrical remodeling". In addition to substantial 


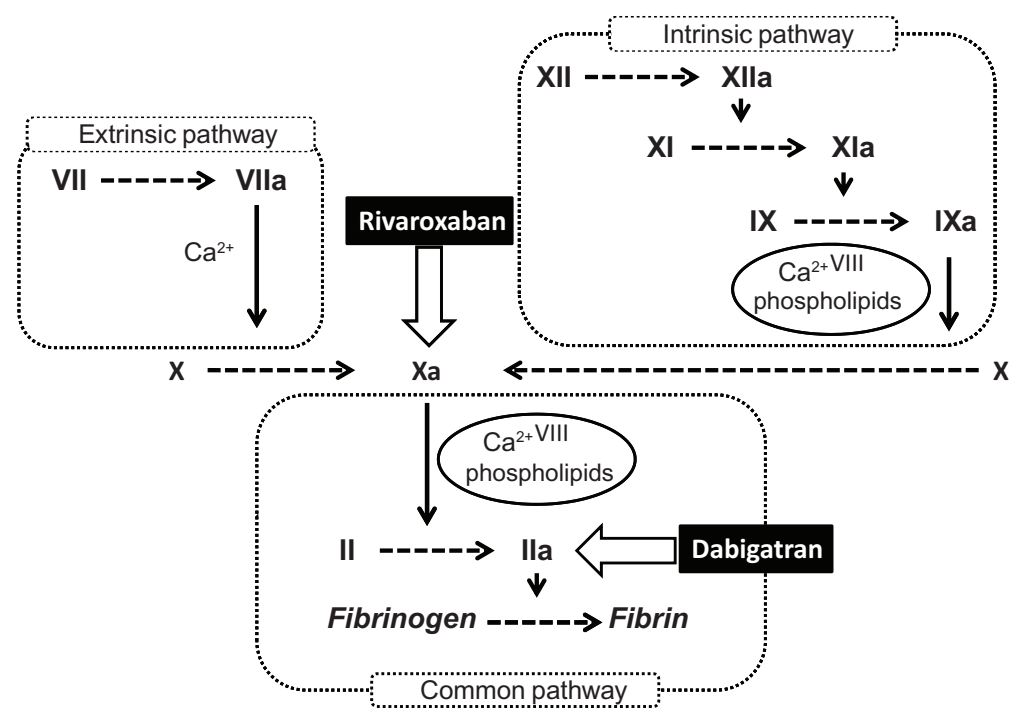

Figure I Activation cascade of the human coagulation system showing mechanisms of action for rivaroxaban and dabigatran. In comparison, warfarin acts by inhibiting synthesis of factors II, VII, IX, and X.

shortening of the atrial action potential and alteration of other electrophysiologic properties, atrial fibrillation also causes significant structural changes ("structural remodeling") in atrial tissue. Electrical and structural remodeling processes take place in atrial myocytes as well as in atrial endocardial cells (the interior surface layer of both upper heart chambers). In order to understand the impact of atrial fibrillation on adhesion molecule expression, one needs to understand these remodeling processes and their impact on downstream processes. Recent studies have provided first insights into the molecular mechanisms involved in the development of cellular and subcellular changes. Altered intracellular calcium ion homeostasis and angiotensin II receptor type 1 activation have been identified as important remodeling factors contributing to cellular and cardiac hypertrophy, atrial extracellular matrix accumulation, and fibrosis in atrial fibrillation. ${ }^{7,9,10}$ Cellular calcium overloading on the one hand and angiotensin II receptor type 1-induced nicotinamide adenine dinucleotide phosphate (NADPH) oxidase activity on the other hand have been demonstrated to cause excessive intracellular oxidative stress. Oxidative stress itself has also been identified to trigger atrial remodeling during atrial fibrillation ("positive feedback loop"). Gene expression profiling of atrial tissue samples from patients with sinus rhythm and atrial fibrillation revealed a decreased expression of antioxidative genes, whereas expression of five reactive oxygen species-producing genes was increased. ${ }^{11-13}$ At the molecular level, several atrial fibrillation-related alterations of atrial tissue are due to activation of different signal transduction systems. Recently, we have been able to show the impact of the nuclear factor kappa B pathway in the process of rapid pacing-induced oxidative stress. ${ }^{11}$ Nuclear factor kappa B in turn leads to upregulation of atrial adhesion molecule expression and reduced nitric oxide levels, which appears to influence the prothrombotic state of the endocardium in fibrillating atria., ${ }^{74}$

\section{Prothrombotic endocardial remodeling}

Thrombus formation within the vascular system depends on pathological endothelial changes. ${ }^{6,7,14,15}$ The remodeling processes mentioned earlier affect myocardial cells and the atrial endothelium ("endocardial remodeling"). Endothelial damage and prothrombotic endothelial alteration are present in fibrillating atria. ${ }^{72}$ They are a prerequisite for the development of atrial clots, because atrial thrombi always start to grow from the atrial wall. Virchow's triad defines circumstances under which thrombus formation is likely. It also applies to atrial thrombus formation during atrial fibrillation. Accordingly, thrombi develop in the presence of reduced blood velocity (circulatory stasis), when the activity of the clotting system is increased (hypercoagulable state), and in the presence of endothelial alterations (endothelial injury). While the loss of regular atrial contractions reduces blood flow velocity, particularly in the atrial appendages, atrial fibrillation is also associated with an activation of the plasma clotting system and of platelets. ${ }^{6,16-19}$ However, the contribution of endothelial alterations to atrial thrombogenesis is not yet fully understood. Numerous studies have shown that atrial fibrillation is associated 
with an inflammatory response measurable by systemic inflammatory markers. ${ }^{13}$ In turn, increased inflammatory markers and increased leukocyte-platelet interactions are predictors of atrial thrombus formation and thromboembolic stroke. ${ }^{20,21}$ Increased endothelial expression of adhesion molecules and reduced levels of nitric oxide could be an important link between the initiation of proinflammatory and prothrombogenic mechanisms responsible for atrial thrombus formation. ${ }^{12,14,18}$ Atrial fibrillation-induced thromboemboli are very leukocyte-rich, showing the close link between coagulation and inflammation in atrial thrombus formation. ${ }^{19}$ In atrial fibrillation, interaction between inflammatory cells and platelets is increased by enhanced platelet P-selectin expression binding to its endothelial receptor P-selectin glycoprotein ligand-1. ${ }^{14,16}$ Kamiyama was also able to show increased P-selectin expression on the endothelial surface layer, which was accompanied by positively intercellular adhesion molecule (ICAM)-1-stained adherent leucocytes in an rabbit atrial fibrillation model. ${ }^{20}$ Interestingly, soluble ICAM-1 and vascular cellular adhesion molecule (VCAM)-1 levels were also found to be elevated in patients with silent cerebral infarction. ${ }^{21}$ It is of note that soluble adhesion molecule levels were found to have a high predictive value with membrane-bound intramyocardial adhesion molecules. ${ }^{22}$ In line with these study results, it was demonstrated that systemic plasma levels of ICAM-1 and VCAM-1 levels are increased in patients with atrial fibrillation, reaching the highest levels in patients with atrial thrombi. Soluble VCAM-1 levels appear to be an independent predictor of atrial thrombi in multivariate analysis of variance. ${ }^{6,15,18,23,24}$ Recently, in ex vivo experiments using organotypic human atrial tissue cultures and in vivo experiments, we found increased atrial expression of VCAM-1 in patients with paroxysmal and persistent atrial fibrillation. Importantly, it was shown that increased expression of atrial adhesion molecules and reduced levels of nitric oxide occur within hours of rapid atrial pacing and is related to increased oxidative stress and generation of reactive oxygen species..$^{12,14,15}$ In addition to age and reduced left ventricular function, soluble VCAM-1 was an independent predictor of mortality in patients with atrial fibrillation. ${ }^{25}$ It is of note that irbesartan and olmesartan therapy reduced adhesion molecule expression in atrial tissue during rapid pacing. Nevertheless, the clinical benefit of angiotensin receptor blocker therapy in reducing thromboembolism in atrial fibrillation has still to be proven. However, the concept of endocardial remodeling in association with complex formation of tissue factor and factor VIIa inducing fibrin generation appears to be very helpful in explaining the constant impact of various diseases like hypertension and diabetes, defined in the $\mathrm{CHADS}_{2}$ score on atrial thrombogenesis (Figure 2). ${ }^{6,7}$

\section{Thrombin and thrombogenesis}

Protease-activated receptors (PAR) 1-4 are the main thrombin receptors, which are expressed on platelets and other cell types including endothelial cells. ${ }^{8}$ Mice lacking PAR-4 are protected from arterial thrombosis, indicating that activation of platelets by thrombin is essential for initiation of the hemostatic process. Accordingly, both PAR-4 and PAR-1 represent promising antithrombotic targets that might provide effective platelet inhibition without the liability of increased bleeding. However, thrombin is not at all, or only marginally, generated in the early phase of arterial thrombus formation and, therefore, an important role for thrombin in initial platelet activation seems unlikely. Instead, tissue factor-induced thrombin accumulation might be important in triggering secretion of platelet granules and the exposure of phosphatidylserine on the platelet membrane. Although tissue factor has a pivotal role in initiating blood coagulation, further activation of coagulation factor $\mathrm{X}$ through the formation of a complex between tissue factor and factor VIIa seems insufficient to support and maintain the next step of blood coagulation. This step coincides with the so-called thrombin burst and involves all coagulation factors of the intrinsic pathway. The low amount of thrombin generated by the extrinsic pathway $(<2 \%)$ is efficiently balanced by the much higher concentration of thrombin generated in the thrombin burst. This mechanism also exerts positive

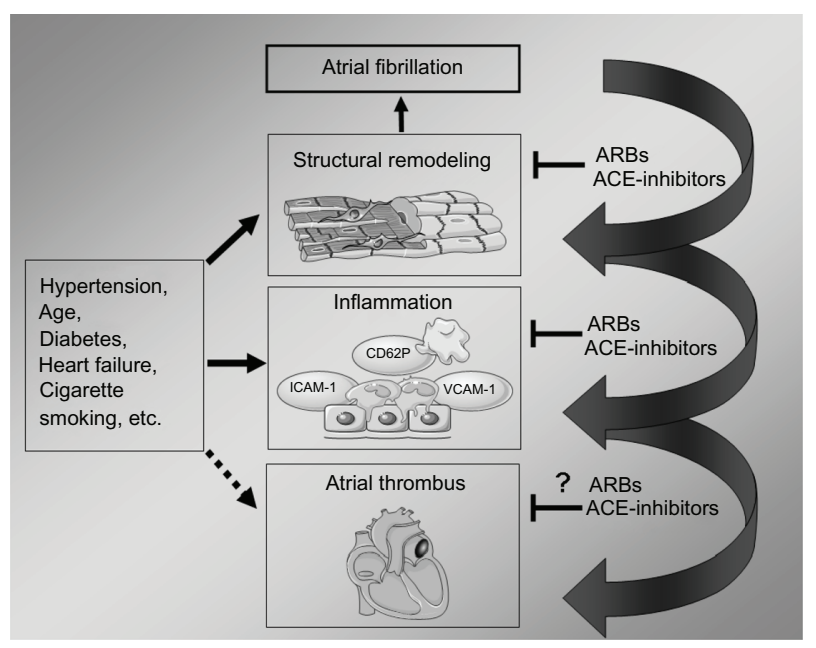

Figure 2 Atrial fibrillation causes structural remodeling and inflammation (red arrows). Upregulation of adhesion molecules amongst other predisposing factors leads to thrombus formation. Angiotensin receptor blockers and angiotensinconverting enzyme inhibitors positively influence remodeling and inflammation. Abbreviation: ARBs, angiotensin recepter blockers. 
feedback on the intrinsic pathway. The thrombin burst is also necessary for the activation of carboxypeptidase B2 (also known as thrombin-activatable fibrinolysis inhibitor), which contributes to the downregulation of plasma fibrinolysis by attenuating plasmin-mediated fibrin degradation. Blood coagulation culminates in the conversion of fibrinogen to fibrin, polymerization of fibrin, and stabilization of the fibrin network. Thrombin-catalyzed cleavage of fibrinopeptide A and fibrinopeptide B converts fibrinogen into fibrin, which spontaneously polymerizes and forms double-stranded protofibrils; these protofibrils then assemble into branched fibrin fibers that form the fibrin clot. Thus, inhibition of thrombin appears as an effective strategy to inhibit thrombus formation and thrombus growth at various stages (Figure 1). ${ }^{8}$

\section{Direct thrombin inhibitors}

Thrombin has three different domains that are possible targets for thrombin-inhibiting drugs, ie, its active site and two anion-binding exosites, ABE-1 and ABE-2. ${ }^{26}$ Direct thrombin inhibitors can bind to the active site and/or ABE-1. Direct thrombin inhibitors binding to the catalytic site and ABE-1 at the same time are called bivalent direct thrombin inhibitors as opposed to substances that only engage the active site (monovalent direct thrombin inhibitors). Compared with heparin, all direct thrombin inhibitors have the advantage that antithrombin-III is not needed as a cofactor, leading to the ability to deactivate both free and clot-bound thrombin. ${ }^{27}$ Furthermore, no direct thrombin inhibitors in use to date have shown relevant plasma protein binding, resulting in a more predictable interindividual response. Due to their lack of interaction with platelet factor IV, direct thrombin inhibitors cannot cause life-threatening heparin-induced thrombocytopenia. ${ }^{28}$ Common direct thrombin inhibitors include the well-known lepirudin, argatroban, bivalirudin, dabigatran, and ximelagatran. In clinical practice, dabigatran and three different factor $\mathrm{Xa}$ inhibitors will be available in the near future to treat patients with atrial fibrillation (Table 1).

\section{Pharmacology of dabigatran}

Dabigatran etexilate (Pradaxa ${ }^{\circledR}$; Boehringer Ingelheim Pharma $\mathrm{GmbH}$ and $\mathrm{Co} \mathrm{KG}$, Ingelheim, Germany) is an oral prodrug, which, after absorption, is converted to dabigatran, the active metabolite, that inhibits thrombin. ${ }^{29,30}$ In vitro studies have shown that dabigatran selectively and reversibly inhibits both free and clot-bound thrombin by binding to the active site of thrombin. ${ }^{30-32}$ Dabigatran shows antithrombotic efficacy in animal models of venous thrombosis, and inhibits thrombin-induced platelet aggregation in vitro. ${ }^{31,33,34}$ After oral administration, dabigatran is absorbed in the intestine. ${ }^{35,36}$ However, absorption of the prodrug is achieved by an acid core of the tablet, which might be a cause of the dyspepsia observed in the clinical trials. Peak plasma concentrations $\left(\mathrm{C}_{\max }\right)$ of dabigatran are achieved approximately 1.5 hours (within 1-2 hours) after oral administration. ${ }^{7,8}$ The bioavailability of dabigatran after oral administration of dabigatran is approximately $6 \%-7 \% .{ }^{35}$ Renal excretion of unchanged dabigatran is the predominant pathway for elimination of dabigatran; about $80 \%$ of an intravenous dose of dabigatran is excreted unchanged in the urine. ${ }^{35}$ In addition, a metabolic pathway involves conjugation with glucuronic acid, resulting in the formation of pharmacodynamically active glucuronides. ${ }^{35}$ These conjugates account for about $20 \%$ of the total dabigatran in plasma. Mean $\mathrm{C}_{\max }$ and area

Table I Overview of key pharmacological properties of novel oral anticoagulants in comparison with dabigatran. Dabigatran is the only direct thrombin inhibitor among the newly available substances and has several specifications

\begin{tabular}{lllll}
\hline & Rivaroxaban & Apixaban & Edoxaban & Dabigatran \\
\hline $\mathrm{T}_{\max }$ & $2-3$ hours & $\mathrm{I}-3$ hours & $\mathrm{I}-3$ hours & I hour \\
Bioavailability & $-40 \%$ & $66 \%$ & $>45 \%$ & $3 \%-7 \%$ \\
Protein binding & $92 \%-95 \%$ & $87 \%$ & $54 \%$ & $35 \%$ \\
CYP metabolism & $32 \%$ & $15 \%$ & $<4 \%$ & 0 \\
Transporter & P-gp/BCRP & P-gP & P-gP & P-gP \\
Half-life & $5-9$ hours & $8-15$ hours & $8-10$ hours & I2-I7 hours \\
Renal excretion & $66 \%$ total & $25 \%-28 \%$ total & $35 \%$ total & $7 \%$ total \\
& $36 \%$ & $24 \%$ & $24 \%$ & Nearly all \\
Fecal & Unchanged & Unchanged & Unchanged & unchanged \\
Dose regimen & $28 \%$ & $56 \%$ & $62 \%$ & $86 \%$ \\
Accumulation factor & QD & BID & QD & BID \\
\hline
\end{tabular}

Notes: It is a prodrug with relatively poor bioavailability and its excretion is mostly renal. The substance does not have interaction with cytochrome P450 metabolism pathways.

Abbreviations: CYP, cytochrome P450; QD, once daily; BID, twice daily; P-gP, P glycoprotein; BCRP, breast cancer resistant protein. 
under the concentration-time curve (AUC) increase in proportion to dose following single oral dabigatran etexilate doses (10-400 mg) and multiple doses (50-400 mg three times daily), demonstrating a linear, dose-proportional pharmacokinetic profile. ${ }^{36}$ Dabigatran has a terminal halflife $\left(t_{1 / 2}\right)$ of about $12-17$ hours (Table 1$) .^{36-38}$ Time curves for assays of antithrombotic activity, including activated partial thromboplastin time and thrombin and ecarin clotting times parallel plasma concentration-time curves, with values increasing rapidly and dose-dependently. The activated partial thromboplastin time assay can provide a useful qualitative assessment of anticoagulant activity but is less sensitive at supratherapeutic dabigatran levels. ${ }^{39}$ Assay oversensitivity with conventional thrombin clotting time methods can be resolved by using the Hemoclot ${ }^{\circledR}$ assay, a precise and sensitive thrombin clotting time method using dabigatran standards, which enables quantitative measurement of dabigatran activity in diluted plasma samples. ${ }^{39}$

\section{Dabigatran and renal function}

Dabigatran levels are increased by renal impairment and correlate with the severity of renal dysfunction. $\mathrm{AUC}_{0-\infty}$ values were shown to be 1.5 -fold, 3.2-fold, and 6.3-fold higher in subjects with mild, moderate and severe renal impairment (creatinine clearance $>50$ to $\leq 80,>30$ to $\leq 50$, and $\leq 30 \mathrm{~mL} /$ minute, respectively) compared with healthy subjects. In patients with end-stage renal disease, the dosenormalized $\mathrm{AUC}_{\infty}$ was approximately twice the value in the control group. Hemodialysis was found to remove $62 \%-68 \%$ of the dabigatran dose from the plasma of patients with endstage renal failure. These findings indicate that a dose reduction and/or an increase in the administration interval may be appropriate in patients with renal impairment (Table 1). ${ }^{40}$

\section{Drug-drug interactions}

Administration of dabigatran etexilate with or without food has no effect on $\mathrm{C}_{\max }$ or AUC of dabigatran, although food reduced the interindividual variability in these parameters and increased the median time to maximum concentration $\left(\mathrm{t}_{\max }\right)$ from 2 to 4 hours in healthy volunteers. ${ }^{38,41}$ Dabigatran is not metabolized by hepatic cytochrome P450 isoenzymes, does not affect the metabolism of other drugs that utilize this system, and has a low potential for drug-drug interactions. ${ }^{42-44}$ Preclinical and clinical studies have shown that dabigatran etexilate, but not dabigatran, is a P-glycoprotein substrate and its bioavailability may be altered by P-glycoprotein inhibitors or inducers. ${ }^{42}$ Studies have shown that drugs that inhibit the efflux transporter, P-glycoprotein, including verapamil, dronedarone, amiodarone, quinidine, and itraconazole, raise serum dabigatran concentrations. ${ }^{42}$

\section{Anticoagulation monitoring}

There is no requirement for routine anticoagulation monitoring when taking dabigatran etexilate. ${ }^{35,36}$ The ecarin clotting time assay displays a linear relationship with drug plasma concentrations in the clinically relevant drug concentration range, and exhibits adequate sensitivity and precision. ${ }^{36}$ The ecarin clotting time assay is not routinely performed in clinical laboratories and, because it has not been standardized or validated for use with dabigatran, it is unsuitable for the quantitative analysis of dabigatran. The International Normalized Ratio (INR) is insensitive to the activity of dabigatran and is therefore unsuitable as a measure of anticoagulant activity. ${ }^{39}$ The thrombin clotting time assay exhibited a linear relationship with plasma concentration with a high level of sensitivity, so the thrombin clotting time assay provides a direct measure of thrombin inhibition and is also highly sensitive to dabigatran. ${ }^{39}$ However, the conventional thrombin clotting time assay is probably too sensitive for routine monitoring, because at dabigatran concentrations $>600 \mathrm{ng} / \mathrm{mL}$, the test often exceeds the maximum measurement times of most laboratory coagulometers. The activated partial thromboplastin time assay is widely available and is useful for providing a qualitative assessment of anticoagulant activity of dabigatran. While activated partial thromboplastin time measurement is not suitable for precise quantification of anticoagulant effect, especially at high plasma concentrations of dabigatran, the activated partial thromboplastin time test may be useful to assist in determining an excess of anticoagulant activity. The Hemoclot ${ }^{\circledR}$ (Hyphen BioMed, Andrésy, France) direct thrombin inhibitor assay with dabigatran calibrators is currently the preferred measure..$^{39}$

\section{Dabigatran in cerebral bleeding models}

Whereas warfarin pretreatment is associated with greater hematoma expansion after intracerebral hemorrhage, the effect direct thrombin inhibitors would have remains unclear. Using different experimental models of intracerebral hemorrhage, the study by Lauer et al compared hematoma volume among dabigatran-treated mice, warfarin-treated mice, and controls. ${ }^{45} \mathrm{CD}-1$ mice were administered dabigatran or warfarin. Sham-treated mice served as controls. At the time point of intracerebral hemorrhage induction, dabigatran mice revealed an increased activated partial thromboplastin time compared with controls, whereas warfarin pretreatment 
resulted in a prothrombin time prolongation. Twenty-four hours after collagenase-induced intracerebral hemorrhage formation, hematoma volume was $3.8 \pm 2.9 \mu \mathrm{L}$ in controls, $4.8 \pm 2.7 \mu \mathrm{L}$ in mice anticoagulated with dabigatranetexilate, and $14.5 \pm 11.8 \mu \mathrm{L}$ in mice treated with warfarin ( $\mathrm{n}=16$, Welch analysis of variance between-group differences, $P=0.007$ ). In addition, a model of laser-induced cerebral microhemorrhage was applied, and the distances that red blood cells and blood plasma were pushed into the brain were quantified. Warfarin mice showed enlarged red blood cells and blood plasma diameters comparable with controls, but no difference was found between dabigatran mice and controls. Lauer et al concluded that in contrast with warfarin, pretreatment with dabigatran did not increase hematoma volume in two different experimental models of intracerebral hemorrhage. Thus, dabigatran might be safer than warfarin even in the setting of cerebral bleed.

\section{Clinical trials of dabigatran}

After demonstrating safety in a multicenter, open-label, dose-escalating study (BISTRO I), dabigatran was evaluated in a multicenter, parallel-group, double-blind study in 1973 patients regarding the incidence of venous thromboembolism after hip or knee replacement as compared with enoxaparin (BISTRO II). ${ }^{46}$ This study could prove dabigatran to be superior at doses of 150 and $225 \mathrm{mg}$ bid. ${ }^{47}$ Subsequently, three larger Phase III studies for the treatment of deep venous thrombosis compared dabigatran (150 mg or $220 \mathrm{mg}$ once daily) with enoxaparin. Two studies (RE-NOVATE and RE-MODEL) showed noninferiority concerning safety and efficacy of dabigatran compared with enoxaparin $40 \mathrm{mg}$ once daily. ${ }^{48,49}$ In another larger study (RE-MOBILIZE), both doses of dabigatran were inferior to a dose of $30 \mathrm{mg}$ enoxaparin twice daily. ${ }^{50}$ However, the differences observed in this study were predominantly due to asymptomatic distal deep venous thromboses with major thromboembolism occurring at similar rates in all treatment groups. In patients with atrial fibrillation, one dose-finding study compared dabigatran $(50,150$, or $300 \mathrm{mg}$ twice daily for 12 weeks) with or without concomitant aspirin ( $80 \mathrm{mg}$ or $325 \mathrm{mg}$ once daily) against warfarin alone (PETRO trial). Aspirin treatment was terminated early because of increased bleeding events observed in the $300 \mathrm{mg}$ dabigatran group (but not in the lower-dose groups studied). Anticoagulant activity in the $150 \mathrm{mg}$ group was equally effective as in the $300 \mathrm{mg}$ or warfarin group. These results suggest that the safety and efficacy profile of dabigatran $150 \mathrm{mg}$ twice daily is comparable with standard warfarin therapy. ${ }^{51}$ Several other trials are underway at the moment, mostly investigating the influence of dabigatran in the context of prevention and therapy of venous thromboembolism (Table 1). Most recently, the results of the RE-LY trial have been published. ${ }^{52-54}$ In this trial, over 18,000 patients with atrial fibrillation received either one of two doses of dabigatran (150 mg or $110 \mathrm{mg}$ ) or adjusted-dose warfarin (mean time in therapeutic range 64\% [median 67\%]). The primary outcome was systemic embolism or stroke. The primary outcome occurred in $1.53 \%$ of the $110 \mathrm{mg}$ dabigatran twice daily group $(P<0.001$ for noninferiority) and in $1.11 \%$ of the $150 \mathrm{mg}$ dabigatran twice daily group $(P<0.001$ for superiority; warfarin 1.69\%). The major bleeding rate in the dabigatran groups was $2.71 \%$ versus $3.11 \%$ in the $110 \mathrm{mg}$ twice daily $(P=0.003)$ and $150 \mathrm{mg}$ twice daily $(P=0.31)$ groups, respectively. Notably, the rate of hemorrhagic stroke was low in both dabigatran arms $(150 \mathrm{mg}, 0.1 \%, P<0.001$; $110 \mathrm{mg}, 0.12 \%, P<0.001$; warfarin, $0.38 \%$ ). In summary, dabigatran given at a dose of $150 \mathrm{mg}$ twice daily prevented more strokes than the $110 \mathrm{mg}$ dose, while the latter caused fewer hemorrhages. The $110 \mathrm{mg}$ dose twice daily was proven noninferior to warfarin while the $150 \mathrm{mg}$ dose twice daily was superior in preventing the primary outcome. Notably, patients with a creatinine clearance of $\leq 30 \mathrm{~mL} /$ minute or liver disease were excluded from the study.

\section{Dabigatran for secondary prevention}

Diener et al assessed the effects of dabigatran compared with warfarin in the subgroup of patients with previous stroke or transient ischemic attack. ${ }^{55}$ Within the subgroup of patients with previous stroke or transient ischemic attack, 1195 patients were from the $110 \mathrm{mg}$ dabigatran twice daily group, 1233 from the $150 \mathrm{mg}$ dabigatran twice daily group, and 1195 from the warfarin group. Stroke or systemic embolism occurred in 65 patients $(2.78 \%$ per year) on warfarin compared with 55 (2.32\% per year) on $110 \mathrm{mg}$ dabigatran twice daily (relative risk [RR]: 0.84 , 95\% confidence interval $[\mathrm{CI}]: 0.58-1.20)$ and 51 (2.07\% per year) on $150 \mathrm{mg}$ dabigatran twice daily (RR: $0.75,95 \% \mathrm{CI}$ : $0.52-1.08)$. The rate of major bleeding was significantly lower in patients on $110 \mathrm{mg}$ dabigatran twice daily (RR: 0.66, 95\% CI: 0.48-0.90) and similar in those on $150 \mathrm{mg}$ dabigatran twice daily (RR: 1.01; 95\% CI: 0.77-1.34) compared with those on warfarin. The effects of both doses of dabigatran compared with warfarin were not significantly different between patients with previous stroke or transient ischemic attack and those without for any of the outcomes from RE-LY apart from vascular death (110 mg group compared with warfarin group, 
interaction $P=0.038$ ). Thus, in patients with previous stroke or transient ischemic attack, the effects of $110 \mathrm{mg}$ dabigatran twice daily and $150 \mathrm{mg}$ dabigatran twice daily on stroke or systemic embolism were similar to those of warfarin. Most effects of both dabigatran doses were consistent in patients as compared with those without previous stroke or transient ischemic attack. ${ }^{55}$

\section{Dabigatran in the elderly}

In the RE-LY trial, there was a significant treatment-byage interaction, such that dabigatran $110 \mathrm{mg}$ twice a day compared with warfarin was associated with a lower risk of major bleeding in patients aged $<75$ years $(1.89 \%$ versus $3.04 \% ; P=0.001)$ and a similar risk in those aged $\geq 75$ years $(4.43 \%$ versus $4.37 \% ; P=0.001$ for interaction), whereas dabigatran $150 \mathrm{mg}$ twice a day compared with warfarin was associated with a lower risk of major bleeding in those aged $<75$ years $(2.12 \%$ versus $3.04 \% ; P=0.001)$ and a trend toward higher risk of major bleeding in those aged $\geq 75$ years $(5.10 \%$ versus $4.37 \% ; P=0.07 ; P$ for interaction $=0.001)$. The interaction with age was evident for extracranial bleeding, but not for intracranial bleeding, with the risk of the latter being consistently reduced with dabigatran compared with warfarin, irrespective of age. The authors of this study concluded that in patients with atrial fibrillation at risk of stroke, both doses of dabigatran compared with warfarin have lower risks of both intracranial and extracranial bleeding in patients aged $<75$ years. In those aged $\geq 75$ years, the intracranial bleeding risk is lower, but the extracranial bleeding risk is similar or higher with both doses of dabigatran compared with warfarin. ${ }^{56}$

\section{Dabigatran for cardioversion of atrial fibrillation}

Within the RE-LY trial, cardioversion on randomized treatment was permitted. Precardioversion transesophageal echocardiography was encouraged, particularly in dabigatran-assigned patients. ${ }^{57}$ Data from before, during, and 30 days after cardioversion were analyzed. A total of 1983 cardioversions were performed in 1270 patients: 647, 672, and 664 in the dabigatran $110 \mathrm{mg}$ twice daily, dabigatran $150 \mathrm{mg}$ twice daily, and warfarin groups, respectively. For dabigatran $110 \mathrm{mg}$ twice daily, dabigatran $150 \mathrm{mg}$ twice daily, and warfarin, transesophageal echocardiography was performed before $25.5 \%, 24.1 \%$, and $13.3 \%$ of cardioversions, of which $1.8 \%, 1.2 \%$, and $1.1 \%$ were positive for left atrial thrombi. Continuous treatment with the study drug for $\geq 3$ weeks before cardioversion was lower on dabigatran
$110 \mathrm{mg}(76.4 \%)$ twice daily and dabigatran $150 \mathrm{mg}$ twice daily $(79.2 \%)$ compared with warfarin $(85.5 \% ; P=0.01$ for both). Stroke and systemic embolism rates at 30 days were $0.8 \%, 0.3 \%$, and $0.6 \%$ (dabigatran $110 \mathrm{mg}$ versus warfarin, $P=0.71$; dabigatran $150 \mathrm{mg}$ versus warfarin, $P=0.40)$ and similar in patients with and without transesophageal echocardiography. Major bleeding rates were $1.7 \%, 0.6 \%$, and $0.6 \%$ (dabigatran $110 \mathrm{mg}$ versus warfarin, $P=0.06 ; 150 \mathrm{mg}$ versus warfarin, $P=0.99$ ). Thus, the authors concluded that the frequencies of stroke and major bleeding within 30 days of cardioversion on the two doses of dabigatran were low and comparable with those on warfarin with or without transesophageal echocardiography guidance. ${ }^{57}$

\section{Dabigatran compared with different INR levels}

Effectiveness and safety of warfarin is associated with the time spent in the therapeutic range, ie, with an international normalized ratio (INR) of 2.0-3.0. In the RE-LY trial, dabigatran versus warfarin reduced both stroke and hemorrhage. ${ }^{58}$ We aimed to investigate the primary and secondary outcomes of the RE-LY trial in relation to each center's mean time in therapeutic range (cTTR) in the warfarin population. For 18,024 patients at 906 sites, the cTTR was estimated by averaging time in therapeutic range for individual warfarin-treated patients calculated by the Rosendaal method. Wallentin et al compared the outcomes of RE-LY across the three treatment groups in four groups defined by the quartiles of cTTR. The authors found that the quartiles of cTTR for patients in the warfarin group were less than $57.1 \%, 57.1 \%-65.5 \%, 65.5 \%-72.6 \%$, and greater than $72.6 \%$. There were no significant interactions between cTTR and prevention of stroke and systemic embolism with either $110 \mathrm{mg}$ dabigatran twice daily (interaction $P=0.89$ ) or $150 \mathrm{mg}$ dabigatran twice daily (interaction $P=0.20$ ) versus warfarin. Neither were any significant interactions recorded for cTTR with regards to intracranial bleeding on $110 \mathrm{mg}$ dabigatran twice daily (interaction $P=0.71$ ) or $150 \mathrm{mg}$ dabigatran twice daily (interaction $P=0.89$ ) versus warfarin. There was a significant interaction between cTTR and major bleeding when comparing $150 \mathrm{mg}$ dabigatran twice daily with warfarin (interaction $P=0.03$ ), with fewer bleeding events at lower cTTR but similar events at higher cTTR, whereas rates of major bleeding were lower with $110 \mathrm{mg}$ dabigatran twice daily than with warfarin irrespective of cTTR. There were significant interactions between cTTR and effects on both $110 \mathrm{mg}$ and $150 \mathrm{mg}$ dabigatran twice daily versus warfarin for the composite of all cardiovascular 
events (interaction $P=0.036$ and $P=0.0006$, respectively) and total mortality (interaction $P=0.066$ and $P=0.052$, respectively), with reduced event rates at low cTTR and similar rates at high cTTR. The authors of this subanalysis concluded that the benefits of dabigatran $150 \mathrm{mg}$ twice daily in reducing stroke, dabigatran $110 \mathrm{mg}$ twice daily at reducing bleeding, and both doses in reducing intracranial bleeding versus warfarin were consistent, irrespective of quality of INR control at the individual centers. For all vascular events, nonhemorrhagic events, and mortality, the advantages of dabigatran were greater at sites with poor INR control than at those with good INR control. Overall, these results show that local standards of care affect the benefits of use of new treatment alternatives. ${ }^{58}$

\section{Dabigatran in warfarin-naïve patients}

In RE-LY, warfarin and two doses of dabigatran, ie, $110 \mathrm{mg}$ twice daily and $150 \mathrm{mg}$ twice daily, were compared in a balanced population of vitamin $\mathrm{K}$ antagonist-naïve ( $\leq 62$ days of lifetime vitamin $\mathrm{K}$ antagonist exposure, with $33 \%$ never prescribed a vitamin $\mathrm{K}$ antagonist) and vitamin $\mathrm{K}$ antagonist-experienced patients with atrial fibrillation. ${ }^{59}$ Typically, among patients who have not been exposed to warfarin prior to starting vitamin $\mathrm{K}$ antagonist treatment, a higher level of discontinuation can be observed, because a relatively high proportion of patients do not tolerate the medication for a variety of reasons (eg, frequent control). For vitamin $\mathrm{K}$ antagonist-naïve and -experienced patients assigned warfarin, time in therapeutic range (INR 2.0-3.0) was $62 \%$ and $67 \%$, respectively. In vitamin $\mathrm{K}$ antagonistnaïve patients, stroke and systemic embolism rates were $1.57 \%, 1.07 \%$, and $1.69 \%$ per year for dabigatran $110 \mathrm{mg}$ twice daily, dabigatran $150 \mathrm{mg}$ twice daily, and warfarin, respectively. Dabigatran $110 \mathrm{mg}$ twice daily was similar to warfarin $(P=0.65)$. Dabigatran $150 \mathrm{mg}$ twice daily was superior $(P=0.005)$. Major bleeding rates were $3.11 \%$, $3.34 \%$, and $3.57 \%$ per year, respectively. Dabigatran $110 \mathrm{mg}$ twice daily and dabigatran $150 \mathrm{mg}$ twice daily were similar to warfarin. Intracranial bleeding rates were $0.19 \%, 0.33 \%$, and $0.73 \%$ per year, respectively. Dabigatran $110 \mathrm{mg}$ twice daily and dabigatran $150 \mathrm{mg}$ twice daily were lower than warfarin $(P=0.001$ and $P=0.005)$. In vitamin $\mathrm{K}$ antagonistexperienced patients, stroke and systemic embolism rates were $1.51 \%, 1.15 \%$, and $1.74 \%$ per year for dabigatran $110 \mathrm{mg}$, dabigatran $150 \mathrm{mg}$, and warfarin, respectively. Dabigatran $110 \mathrm{mg}$ twice daily was similar to warfarin $(P=0.32)$; dabigatran $150 \mathrm{mg}$ twice daily was superior $(P=0.007)$.
Major bleeding rates were $2.66 \%, 3.30 \%$, and 3.57\% per year, respectively. Dabigatran $110 \mathrm{mg}$ twice daily was lower than warfarin $(P=0.003)$; dabigatran $150 \mathrm{mg}$ twice daily was similar $(P=0.41)$. Intracranial bleeding rates were $0.26 \%$, $0.32 \%$, and $0.79 \%$ per year, respectively. Dabigatran $110 \mathrm{mg}$ twice daily and dabigatran $150 \mathrm{mg}$ twice daily were lower than warfarin $(P=0.001$ for both). Results were similar for patients never on a vitamin $\mathrm{K}$ antagonist. Thus, the conclusion of this study was that previous vitamin $\mathrm{K}$ antagonist exposure does not influence the benefits of dabigatran at either dose compared with warfarin. ${ }^{59}$

\section{Cost-effectiveness of dabigatran}

On the basis of the results from RE-LY and other trials, Shah and Gage developed a decision-analysis model to compare the cost and quality-adjusted survival of various antithrombotic therapies. ${ }^{60}$ Shah and Gage ran a Markov model in a hypothetical cohort of 70-year-old patients with atrial fibrillation using a cost-effectiveness threshold of $\$ 50,000 /$ quality-adjusted life-year. The authors estimated the cost of dabigatran as US\$9 a day. For a patient with an average risk of major hemorrhage (about 3\% per year), the most cost-effective therapy depended on stroke risk. For patients with the lowest stroke risk $\left(\mathrm{CHADS}_{2}\right.$ stroke score 0$)$, only aspirin was cost-effective. For patients with a moderate stroke risk (CHADS 2 score 1-2), warfarin was cost-effective unless the risk of hemorrhage was high or quality of INR control was poor (time in the therapeutic range $<57.1 \%$ ). For patients with a high stroke risk $\left(\mathrm{CHADS}_{2}\right.$ stroke score $\geq 3$ ), dabigatran $150 \mathrm{mg}$ twice daily was cost-effective unless INR control was excellent (time in the therapeutic range $>72.6 \%$ ). Neither dabigatran $110 \mathrm{mg}$ nor dual therapy (aspirin and clopidogrel) was cost-effective. Thus, in that study, dabigatran $150 \mathrm{mg}$ twice daily was cost-effective in populations at high risk of hemorrhage or stroke unless INR control with warfarin was excellent. However, warfarin was cost-effective in moderate-risk populations as well unless INR control was poor. ${ }^{60}$

\section{Dabigatran: recent update of guidelines}

Recommendation for Emerging Antithrombotic Agents 2011 Focused Update Recommendation Comments from the American Heart Association/ACC: ${ }^{61}$

Class I - 1. Dabigatran is useful as an alternative to warfarin for the prevention of stroke and systemic thromboembolism in patients with paroxysmal to permanent and risk factors for stroke or systemic embolization who 
do not have a prosthetic heart valve or hemodynamically significant valve disease, severe renal failure (creatinine clearance $<15 \mathrm{~mL} /$ minute), or advanced liver disease (impaired baseline clotting function, level B evidence).

The Canadian guidelines have already implemented a reference to dabigatran, stating that when "oral anticoagulation is indicated most patients should receive dabigatran in preference to warfarin". ${ }^{62}$ They further suggest that "in general the dose of dabigatran $150 \mathrm{mg}$ bid is preferable to a dose of $110 \mathrm{mg}$ bid". The European guidelines suggest its use, and provide guidance with respect to dosage. They recommend that if a patient is at "low risk of bleeding, dabigatran 150 bid may be considered", but if a patient has a measurable risk of bleeding (eg, HASBLED score $>3$ ) dabigatran etexilate $110 \mathrm{mg}$ may be considered. ${ }^{63}$

\section{Comparison with other novel anticoagulants}

At present, the US Food and Drug Administration (FDA) has only reached an additional decision on rivaroxaban regarding other novel anticoagulants. ${ }^{64,65}$ According to FDA policy, it is essential that a new therapy must be as effective as alternatives that are already approved for marketing when the disease to be treated is life-threatening or capable of causing irreversible morbidity. Rivaroxaban was studied in the ROCKET trial, in which 14,000 patients were randomly treated with rivaroxaban $20 \mathrm{mg}$ once daily or warfarin. The ROCKET trial aimed to determine that rivaroxaban was noninferior to warfarin. ROCKET enrolled higher risk patients compared with RE-LY. The study showed in different analyses with regard to the primary endpoint that rivaroxaban is superior to warfarin. However, the FDA questioned some aspects of the trial data. The INR for patients in the warfarin group was in the therapeutic range only $55 \%$ of the time. When the FDA analyzed data only from study sites where patients' average time in the therapeutic range was above specified thresholds, they found that the RR of stroke or systemic embolism with rivaroxaban was near unity compared with warfarin. Interestingly, there was a high rate of stroke and systemic embolism in the rivaroxaban group between day 2 and day 7 after discontinuation of therapy. Therefore, low time in therapeutic range in the warfarin group and exclusion of excess events after treatment discontinuation might have contributed to the favorable results obtained in ROCKET-AF. The FDA noted that the once-daily dosage was not well supported by the pharmacokinetic data, but finally approved the substance for use in patients to "prevent stroke in people with common type of abnormal heart rhythm". It was suggested that rivaroxaban might be used in patients who have a inadequate response to or cannot take dabigatran or warfarin. However, head-to-head comparisons of novel anticoagulants are missing. ${ }^{65}$ Interestingly, and in contrast with previous trials in patients with acute coronary syndromes, rivaroxaban therapy reduced total mortality at a very low dose (2.5 mg twice daily) in the ATLAS ACS2 TIMI 51 trial. The trial results are impressive. Therefore, it might be speculated that new agents may have substantial different effects in various clinical settings. ${ }^{66}$

\section{Conclusion}

Patients at risk of thromboembolic stroke must be assessed on an individual basis, weighing bleeding risk against that of stroke. Dabigatran studied at two doses in the RE-LY trial proved noninferior to warfarin in respect to stroke or systemic embolism. Regarding bleeding events, dabigatran $110 \mathrm{mg}$ twice daily was superior to a vitamin $\mathrm{K}$ antagonist, while at $150 \mathrm{mg}$ twice daily, a higher efficacy in terms of stroke and systemic embolism prevention went along with a risk comparable with that of warfarin. Nevertheless, very recent reports suggest that bleeding and death may occur if dabigatran is used in patients with significant renal impairment. Therefore, renal function has to be carefully evaluated before the initiation of therapy in patients with atrial fibrillation.

\section{Disclosure}

AG has received speaker fees from Boehringer Ingelheim.

\section{References}

1. Go AS, Hylek EM, Phillips KA, et al. Prevalence of diagnosed atrial fibrillation in adults: national implications for rhythm management and stroke prevention: the AnTicoagulation and Risk Factors in Atrial Fibrillation (ATRIA) Study. JAMA. 2001;285:2370-2375.

2. Wolf PA, Dawber TR, Thomas HE Jr, Kannel WB. Epidemiologic assessment of chronic atrial fibrillation and risk of stroke: the Framingham study. Neurology. 1978;28:973-977.

3. Hammwöhner M, Goette A. Will warfarin soon be passe? New approaches to stroke prevention in atrial fibrillation. $J$ Cardiovasc Pharmacol. 2008;52:18-27.

4. Wyse DG, Waldo AL, DiMarco JP, et al. A comparison of rate control and rhythm control in patients with atrial fibrillation. $N$ Engl J Med 2002;347:1825-1833.

5. Blackshear JL, Safford RE. AFFIRM and RACE trials: implications for the management of atrial fibrillation. Card Electrophysiol Rev. 2003;7: 366-369.

6. Hammwöhner M, D'Alessandro A, Wolfram O, Goette A. New pharmacologic approaches to prevent thromboembolism in patients with atrial fibrillation. Curr Vasc Pharmacol. 2007;5:211-219.

7. Schotten U, Verheule S, Kirchhof P, Goette A. Pathophysiological mechanisms of atrial fibrillation: a translational appraisal. Physiol Rev. 2011;91:265-325.

8. Lippi G, Franchini M, Targher G. Arterial thrombus formation in cardiovascular disease. Nat Rev Cardiol. 2011;8:502-512. 
9. Ausma J, Wijffels M, Thone F, Wouters L, Allessie M, Borgers M. Structural changes of atrial myocardium due to sustained atrial fibrillation in the goat. Circulation. 1997;96:3157-3163.

10. Goette A, Honeycutt C, Langberg JJ. Electrical remodeling in atrial fibrillation. Time course and mechanisms. Circulation. 1996;94: 2968-2974.

11. Bukowska A, Schild G, Keilhoff D, et al. Mitochondrial dysfunction and redox signaling in atrial tachyarrhythmia. Exp Biol Med (Maywood). 2008;233:558-574.

12. Cai H, Li Z, Goette A, et al. Downregulation of endocardial nitric oxide synthase expression and nitric oxide production in atrial fibrillation: potential mechanisms for atrial thrombosis and stroke. Circulation. 2002;106:2854-2858.

13. Conway DS, Buggins P, Hughes E, Lip GY. Relationship of interleukin-6 and C-reactive protein to the prothrombotic state in chronic atrial fibrillation. J Am Coll Cardiol. 2004;43:2075-2082.

14. Minamino T, Kitakaze M, Sanada S, et al. Increased expression of $\mathrm{P}$-selectin on platelets is a risk factor for silent cerebral infarction in patients with atrial fibrillation: role of nitric oxide. Circulation. 1998;98: 1721-1727.

15. Goette A, Bukowska A, Lendeckel U, et al. Angiotensin II receptor blockade reduces tachycardia-induced atrial adhesion molecule expression. Circulation. 2008;117:732-742.

16. Goette A, Ittenson A, Hoffmanns P, et al. Increased expression of P-selectin in patients with chronic atrial fibrillation. Pacing Clin Electrophysiol. 2000;23:1872-1875.

17. Goette A, Staack T, Rocken C, et al. Increased expression of extracellular signal-regulated kinase and angiotensin-converting enzyme in human atria during atrial fibrillation. J Am Coll Cardiol. 2000;35:1669-1677.

18. Hammwöhner M, Ittenson A, Dierkes J, et al. Platelet expression of CD40/CD40 ligand and its relation to inflammatory markers and adhesion molecules in patients with atrial fibrillation. Exp Biol Med (Maywood). 2007;232:581-589.

19. Hammwöhner M, Tautenhahn J, Kuester D, Goette A. Inadvertent catch of a factor VIII rich thrombus with a femoral sheath during cardiac catheterization. Int J Cardiol. 2008;130:e39-e41.

20. Kamiyama N. Expression of cell adhesion molecules and the appearance of adherent leukocytes on the left atrial endothelium with atrial fibrillation: rabbit experimental model. Jpn Circ J. 1998;62:837-843.

21. Kawamura T, Umemura T, Kanai A, et al. Soluble adhesion molecules and C-reactive protein in the progression of silent cerebral infarction in patients with type 2 diabetes mellitus. Metabolism. 2006;55: 461-466.

22. Noutsias M, Hohmann C, Pauschinger M, et al. sICAM-1 correlates with myocardial ICAM-1 expression in dilated cardiomyopathy. Int J Cardiol. 2006;91:153-161.

23. Kim YM, Guzik TJ, Zhang YH, et al. A myocardial Nox2 containing $\mathrm{NAD}(\mathrm{P}) \mathrm{H}$ oxidase contributes to oxidative stress in human atrial fibrillation. Circ Res. 2005;97:629-636.

24. Sohara H, Amitani S, Kurose M, Miyahara K. Atrial fibrillation activates platelets and coagulation in a time-dependent manner: a study in patients with paroxysmal atrial fibrillation. J Am Coll Cardiol. 1997;29: 106-112.

25. Ehrlich JR, Kaluzny M, Baumann S, Lehmann R, Hohnloser SH. Biomarkers of structural remodelling and endothelial dysfunction for prediction of cardiovascular events or death in patients with atrial fibrillation. Clin Res Cardiol. 2011;100:1029-1036.

26. Di Nisio M, Klerk CP, Meijers JC, Buller HR. The prognostic value of the D-dimer test in cancer patients treated with and without lowmolecular-weight heparin. J Thromb Haemost. 2005;3:1531-1533.

27. Weitz JI, Hudoba M, Massel D, Maraganore J, Hirsh J. Clot-bound thrombin is protected from inhibition by heparin-antithrombin III but is susceptible to inactivation by antithrombin III-independent inhibitors. J Clin Invest. 1990;86:385-391.

28. Weitz JI, Bates SM. New anticoagulants. J Thromb Haemost. 2005;3: 1843-1853.
29. Di Nisio M, Middeldorp S, Buller HR. Direct thrombin inhibitors. N Engl J Med. 2005;353:1028-1040.

30. Hauel NH, Nar H, Priepke H, Ries U, Stassen JM, Wienen W. Structurebased design of novel potent nonpeptide thrombin inhibitors. $J$ Med Chem. 2002;45:1757-1766.

31. Wienen W, Stassen JM, Priepke H, Ries UJ, Hauel N. In-vitro profile and ex-vivo anticoagulant activity of the direct thrombin inhibitor dabigatran and its orally active prodrug, dabigatran etexilate. Thromb Haemost. 2007;98:155-162.

32. Sanford M, Plosker GL. Dabigatran etexilate. Drugs. 2008;68: 1699-1709.

33. Wienen W, Stassen JM, Priepke H, Ries UJ, Hauel N. Effects of the direct thrombin inhibitor dabigatran and its orally active prodrug, dabigatran etexilate, on thrombus formation and bleeding time in rats. Thromb Haemost. 2007;98:333-338.

34. Wienen W, Stassen JM, Priepke H, Ries UJ, Hauel N. Antithrombotic and anticoagulant effects of the direct thrombin inhibitor dabigatran, and its oral prodrug, dabigatran etexilate, in a rabbit model of venous thrombosis. J Thromb Haemost. 2007;5:1237-1242.

35. Blech S, Ebner T, Ludwig-Schwellinger E, Stangier J, Roth W. The metabolism and disposition of the oral direct thrombin inhibitor, dabigatran, in humans. Drug Metab Dispos. 2008;36:386-399.

36. Stangier J, Rathgen K, Stahle H, Gansser D, Roth W. The pharmacokinetics, pharmacodynamics and tolerability of dabigatran etexilate, a new oral direct thrombin inhibitor, in healthy male subjects. Br J Clin Pharmacol. 2007;64:292-303.

37. Stangier J, Stahle H, Rathgen K, Fuhr R. Pharmacokinetics and pharmacodynamics of the direct oral thrombin inhibitor dabigatran in healthy elderly subjects. Clin Pharmacokinet. 2008;47:47-59.

38. Stangier J, Eriksson BI, Dahl OE, et al. Pharmacokinetic profile of the oral direct thrombin inhibitor dabigatran etexilate in healthy volunteers and patients undergoing total hip replacement. J Clin Pharmacol. 2005;45:555-563.

39. Van Ryn J, Stangier J, Haertter S, et al. Dabigatran etexilate - a novel, reversible, oral direct thrombin inhibitor: interpretation of coagulation assays and reversal of anticoagulant activity. Thromb Haemost. 2010; 103:1116-1127.

40. Stangier J, Rathgen K, Stahle H, Mazur D. Influence of renal impairment on the pharmacokinetics and pharmacodynamics of oral dabigatran etexilate: an open-label, parallel-group, single-centre study. Clin Pharmacokinet. 2010;49:259-268.

41. Stangier J. Clinical pharmacokinetics and pharmacodynamics of the oral direct thrombin inhibitor dabigatran etexilate. Clin Pharmacokinet. 2008;47:285-295

42. Stangier J, Clemens A. Pharmacology, pharmacokinetics, and pharmacodynamics of dabigatran etexilate, an oral direct thrombin inhibitor. Clin Appl Thromb Hemost. 2009;15 (Suppl 1):9S-16S.

43. Stangier J, Rathgen K, Stahle H, Reseski K, Kornicke T, Roth W. Coadministration of dabigatran etexilate and atorvastatin: assessment of potential impact on pharmacokinetics and pharmacodynamics. Am J Cardiovasc Drugs. 2009;9:59-68.

44. Ufer M. Comparative efficacy and safety of the novel oral anticoagulants dabigatran, rivaroxaban and apixaban in preclinical and clinical development. Thromb Haemost. 2010;103:572-585.

45. Lauer A, Cianchetti FA, Van Cott EM, et al. Anticoagulation with the oral direct thrombin inhibitor dabigatran does not enlarge hematoma volume in experimental intracerebral hemorrhage. Circulation. 2011;124:1654-1662.

46. Eriksson BI, Dahl OE, Ahnfelt L, et al. Dose escalating safety study of a new oral direct thrombin inhibitor, dabigatran etexilate, in patients undergoing total hip replacement: BISTRO I. J Thromb Haemost. 2004;2:1573-1580.

47. Eriksson BI, Dahl OE, Buller HR, et al. A new oral direct thrombin inhibitor, dabigatran etexilate, compared with enoxaparin for prevention of thromboembolic events following total hip or knee replacement: the BISTRO II randomized trial. J Thromb Haemost. 2005;3:103-111. 
48. Eriksson BI, Dahl OE, Rosencher N, et al. Oral dabigatran etexilate vs subcutaneous enoxaparin for the prevention of venous thromboembolism after total knee replacement: the RE-MODEL randomized trial. J Thromb Haemost. 2007;5:2178-2185.

49. Eriksson BI, Dahl OE, Rosencher N, et al. Dabigatran etexilate versus enoxaparin for prevention of venous thromboembolism after total hip replacement: a randomised, double-blind, non-inferiority trial. Lancet. 2007;370:949-956.

50. Ginsberg JS, Davidson BL, Comp PC, et al. Oral thrombin inhibitor dabigatran etexilate vs North American enoxaparin regimen for prevention of venous thromboembolism after knee arthroplasty surgery. J Arthroplasty. 2009;24:1-9.

51. Ezekowitz MD, Reilly PA, Nehmiz G, et al. Dabigatran with or without concomitant aspirin compared with warfarin alone in patients with nonvalvular atrial fibrillation (PETRO Study). Am J Cardiol. 2007;100:1419-1426.

52. Ezekowitz MD, Connolly S, Parekh A, et al. Rationale and design of RE-LY: randomized evaluation of long-term anticoagulant therapy, warfarin, compared with dabigatran. Am Heart J. 2009;157:805-810.

53. Connolly SJ, Ezekowitz MD, Yusuf S, et al. Dabigatran versus warfarin in patients with atrial fibrillation. N Engl J Med. 2009;361:1139-1151.

54. Connolly SJ, Ezekowitz MD, Yusuf S, et al. Newly identified events in the RE-LY trial. $N$ Engl J Med. 2010;363:1875-1876.

55. Diener HC, Connolly SJ, Ezekowitz MD, et al; RE-LY study group. Dabigatran compared with warfarin in patients with atrial fibrillation and previous transient ischaemic attack or stroke: a subgroup analysis of the RE-LY trial. Lancet Neurol. 2010;9:1157-1163.

56. Eikelboom JW, Wallentin L, Connolly SJ, et al. Risk of bleeding with 2 doses of dabigatran compared with warfarin in older and younger patients with atrial fibrillation: an analysis of the randomized evaluation of long-term anticoagulant therapy (RE-LY) trial. Circulation. 2011;123:2363-2372.

57. Nagarakanti R, Ezekowitz MD, Oldgren J, et al. Dabigatran versus warfarin in patients with atrial fibrillation: an analysis of patients undergoing cardioversion. Circulation. 2011;123:131-136.
58. Wallentin L, Yusuf S, Ezekowitz MD, et al. Efficacy and safety of dabigatran compared with warfarin at different levels of international normalised ratio control for stroke prevention in atrial fibrillation: an analysis of the RE-LY trial. Lancet. 2010;376:975-983.

59. Ezekowitz MD, Wallentin L, Connolly SJ, et al. Dabigatran and warfarin in vitamin $\mathrm{K}$ antagonist-naive and -experienced cohorts with atrial fibrillation. Circulation. 2010;122:2246-2253.

60. Shah SV, Gage BF. Cost-effectiveness of dabigatran for stroke prophylaxis in atrial fibrillation. Circulation. 2011;123:2562-2570.

61. Wann LS, Curtis AB, Ellenbogen KA, et al. 2011 ACCF/AHA/HRS focused update on the management of patients with atrial fibrillation (update on Dabigatran): a report of the American College of Cardiology Foundation/American Heart Association Task Force on practice guidelines. Circulation. 2011;123:1144-1150.

62. Cairns JA, Connolly S, McMurtry S, et al. Canadian Cardiovascular Society atrial fibrillation guidelines 2010: prevention of stroke and systemic thromboembolism in atrial fibrillation and flutter. Can $J$ Cardiol. 2011;27:74-90.

63. Camm J, Kirchhof P, Lip GY, et al. Guidelines for the management of atrial fibrillation:the Task Force for the Management of Atrial Fibrillation of the European Society of Cardiology (ESC). Eur Heart J. 2010;31:2369-2429.

64. Patel MR, Mahaffey KW, Garg J, et al; ROCKET AF Investigators. ROCKET AF trial. Rivaroxaban versus warfarin in nonvalvular atrial fibrillation. $N$ Engl J Med. 2011;365:883-891.

65. Fleming TR, Emerson SS. Evaluating rivaroxaban for nonvalvular atrial fibrillation - regulatory considerations. N Engl J Med. 2011;365: 1557-1559.

66. Mega J, Braunwald E, Wiviott SD, et al. Rivaroxaban in patients with a recent acute coronary syndrome. $N$ Engl J Med. 2011. doi: 10.1056/ NEJMoa1112277.
Research Reports in Clinical Cardiology

\section{Publish your work in this journal}

Research Reports in Clinical Cardiology is an international, peerreviewed, open access journal publishing original research, reports, editorials, reviews and commentaries on all areas of cardiology in the clinic and laboratory. The manuscript management system is completely online and includes a very quick and fair peer-review system.

\section{Dovepress}

Visit http://www.dovepress.com/testimonials.php to read real quotes from published authors. 